\title{
The research on grid ionospheric delay algorithm of BDSBAS
}

\author{
Geng $\mathrm{Yu}^{1, \mathrm{a}}$, Mo Peng, $\mathrm{b}$ \\ ${ }^{1}$ School of Civil Aviation, Shenyang Aerospace University, Shenyang 110136, China; \\ ${ }^{2}$ School of Electronic Information Engineering, Shenyang Aerospace University, Shenyang 110136, \\ China. \\ ayug@sau.edu.cn, bpengmo@126.com
}

\begin{abstract}
With the development of BD navigation system, it is pressing to develop Satellite-Based Augmentation System of BD system. Based on the observation data on March 20, 2016 of 10 GNSS reference stations from $\mathrm{Cmonoc}$, the paper verifies the accuracy of grid ionospheric correction algorithm of BDSBAS in part of stations in China. The paper compares grid ionospheric model with Klobuchar model, and tests the error of grid ionospheric vertical delay GIVE. The results show that correction effect of grid ionospheric model is superior to that of Klobuchar model and the correction error is in a normal range, so it is workable to apply BD grid ionospheric model in the area of China.
\end{abstract}

Keywords: BD navigation system; Satellite-Based Augmentation System (SBAS); Lonospheric error; Grid ionospheric delay algorithm.

\section{Introduction}

Satellite-Based Augmentation System is a satellite navigation system to enhance the system of the original, through the calculation of the ground about GPS integrity and correction data, and the use of satellites in geosynchronous orbit (GEO) to broadcast signal integrity, data revisions and changes to SBAS users to improve the navigation accuracy and integrity. Many space powers are competing to develop their own satellite based augmentation system, and the Satellite-Based Augmentation System based on the Beidou system is also in the process of preparing for the test. And the ionospheric delay has undoubtedly become an important error source in the BDSBAS implementation process. Satellite-Based Augmentation System is different from the ground-based augmentation system (GBAS), the error correction of ephemeris error, satellite clock error, ionospheric delay and other atmospheric delay errors are computed separately. The users who can receive SBAS message compute ionospheric delay with grid ionospheric delay algorithm, and the users of single frequency who cannot receive SBAS signal use Klobuchar model to correct ionospheric delay.

\section{The principle of SBAS grid ionospheric delay correction}

The average altitude of the ionosphere is $375 \mathrm{~km}$. Therefore, in Beidou system, the ionization level which is $375 \mathrm{~km}$ to ground is used as the common ionospheric reference surface.

Steps to calculate the ionospheric delay are as follows:

(1) The reference plane according to the longitude and latitude interval is divided into a certain number of grid, and the SBAS system broadcasts the ionospheric delay error based on the grid nodes.

(2) Reference station using dual frequency receiver calculates the penetration point of the ionospheric delay value, and calculate the latitude and longitude of Beidou satellite in the ionosphere on the reference plane of the penetration point. These data are sent to the master station through the communication link.

(3) According to the terms of reference station data, master stations calculate vertical delay value of the ionosphere of each grid node on the reference plane, and generate a message according to a certain format and broadcast to users within the region by the synchronous communication satellites.

(4) By calculating the latitude and longitude of the penetration point and comparing the ionospheric delay data of the grid nodes, the ionospheric delay values are obtained according to a certain algorithm. 


\subsection{Calculation task of reference station}

(1)Calculation of the delay value of the point of penetration

Because the ionosphere is a kind of dispersion medium related to the frequency of electromagnetic wave, the user's dual frequency receiver can make the real-time measurement of the ionospheric delay without any mathematical model [1]. If $\rho_{1}$ and $\rho_{2}$ represent the pseudorange measurements of the carrier B1I and B2I which are launched by a dual band receiver at the same time on the same satellite, then, according to the function relationship between the ionosphere delay and the carrier frequency, the ionospheric delay can be obtained:

$$
I_{1}=\frac{f_{2}^{2}}{f_{1}^{2}-f_{2}^{2}}\left(\rho_{2}-\rho_{1}\right)=1.48717\left(\rho_{2}-\rho_{1}\right)
$$

Where, $I_{1}$ is behalf of the ionospheric delay on the frequency of carrier B1I.

(2) Determination of the penetration point

Considering the position relation between the satellite and the reference stations, the reference stations must first determine the location of the ionospheric penetration point in the signal transmission path. The ionospheric penetration point (IPP) is defined as the intersection of the line from the reference station to the satellite and the ionosphere reference plane. The following formula provides a way to calculate the latitude and longitude of the penetration point. First, the latitude is calculated by the following formula:

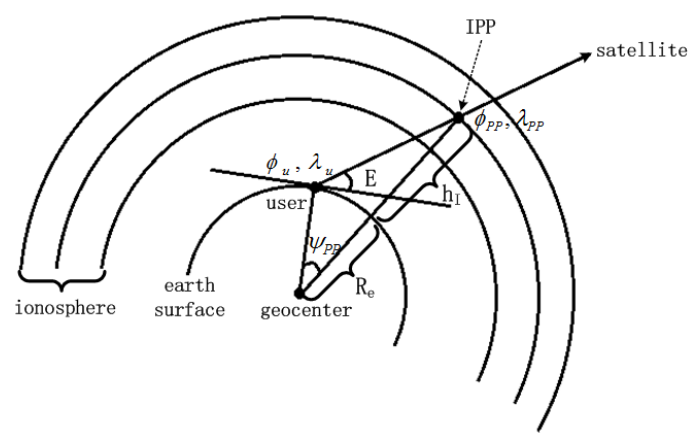

Fig. 1 Geometry of ionospheric pierce point

$\phi_{P P}=\sin ^{-1}\left(\sin \phi_{u} \cos \psi_{P P}+\cos \phi_{u} \sin \psi_{P P} \cos A\right)$

Where, $\psi_{P P}$ represents the angle between the lines from reference station to the center of the earth and the line from the satellite to the center of the earth:

$$
\psi_{P P}=\frac{\pi}{2}-E-\sin ^{-1}\left(\frac{R_{e}}{R_{e}+h_{I}} \cos E\right)
$$

$\mathrm{A}$ is the azimuth angle of the satellite relative to the position of the reference station. $\mathrm{E}$ is the elevation angle of the satellite relative to the reference station. $R_{e}$ is approximate radius of the earth ellipsoid $(6378.1363 \mathrm{~km}) . h_{I}$ is height of the maximum electron density $(375 \mathrm{~km})$. So the longitude of the penetration point is:

$$
\lambda_{P P}=\lambda_{u}+\pi-\sin ^{-1}\left(\frac{\sin \psi_{P P} \sin A}{\cos \phi_{P P}}\right)
$$

(3) The vertical ionospheric delay of the penetration point

The reference station determines the point of penetration delay, which can be divided by the inclination factor $F_{P P}$ to get the vertical delay information $\left(\tau_{v p p}\right)$ of the ionospheric penetration point according to the following formula:

$$
\tau_{v p p}\left(\lambda_{p p}, \phi_{p p}\right)=\frac{I C_{i}}{F_{p p}}
$$

$I C_{i}$ is inclination delay of the ionospheric penetration point. Inclination factor is calculated as follows: 


$$
F_{p p}=\left[1-\left(\frac{R_{e} \cos E}{R_{e}+h_{I}}\right)^{2}\right]^{-\frac{1}{2}}
$$

\subsection{The calculation task of the master station}

The master station of SBAS receives the real time data from all reference station about the ionosphere in the coverage area of SBAS (including the latitude and longitude of the ionospheric penetration point of the reference station, the vertical ionospheric delay of the penetration point).A distance weighted algorithm is used to estimate the vertical ionospheric delay error of the grid nodes in the entire coverage area. The calculation formula is detailed in reference [7].

\subsection{User ionospheric delay error algorithm}

In the SBAS system, the method of user computing the ionospheric delay error is similar to the method of master station computing method according to reference station data to calculate. Only the user calculates the ionospheric delay data based on the source of synchronous satellite broadcasting grid network ionospheric delay information. The specific process is as follows:

(1) Determination of the penetration point

The determination of the penetration point is similar to the reference station. The specific formula is like formula (2), (3), (4). The location of the reference station in the formula is replaced by the user's location.

(2) The determination of the vertical delay of the penetration point of the user

The location of the user penetration point (IPP) is usually different from that of the ionospheric grid (IGP). Therefore, it is necessary for the user to interpolate the IGP delay data, to obtain the data of IPP delay. According to the number of grid points available in the vicinity of the penetration point, we can choose four point interpolation method or three point interpolation method. The principle of the four point interpolation method is as follows:

$$
\tau_{v p p}\left(\phi_{p p}, \lambda_{p p}\right)=\sum_{i=1}^{4} W_{i}\left(x_{p p}, y_{p p}\right) \tau_{v i}
$$

Where, $\tau_{v i}$ is vertical ionospheric delay of four grid points of broadcasting, as is shown in figure 2 .

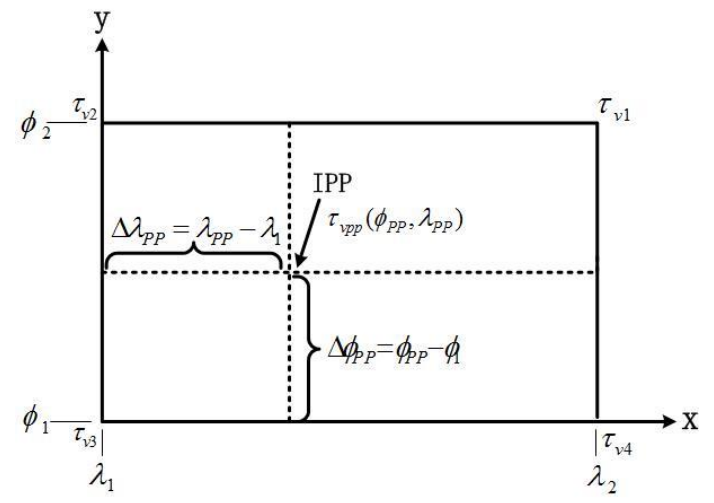

Fig. 2 Four-point interpolation algorithm definitions

Specific weighting functions are as follows:

$$
\begin{aligned}
& W_{1}=x_{p p} y_{p p} \\
& W_{2}=\left(1-x_{p p}\right) y_{p p} \\
& W_{3}=\left(1-x_{p p}\right)\left(1-y_{p p}\right) \\
& W_{4}=x_{p p}\left(1-y_{p p}\right) \\
& x_{p p}=\frac{\Delta \lambda_{p p}}{\lambda_{2}-\lambda_{1}} \\
& y_{p p}=\frac{\Delta \phi_{p p}}{\phi_{2}-\phi_{1}} \\
& \Delta \lambda_{p p}=\lambda_{p p}-\lambda_{1}
\end{aligned}
$$




$$
\Delta \phi_{p p}=\phi_{p p}-\phi_{1}
$$

(3) Determination of the delay value of the user's IPP

After determining the vertical delay of the user penetration point, it can be multiplied inclination factor $F_{P P}$, to obtain ionospheric correction information $I C_{i}$ and add to the calculation of the pseudo range:

$$
I C_{i}=F_{p p} \bullet \tau_{v p p}\left(\lambda_{p p}, \phi_{p p}\right)
$$

\section{Klobuchar ionospheric delay function model}

Single frequency receiver usually uses Klobuchar model to correct ionospheric delay. The Klobuchar model for calculating the Ionospheric Vertical delay correction (unit s) for the B1I signal is specific as follows:

$$
I_{Z}^{\prime}(t)=\left\{\begin{array}{c}
5 \times 10^{-9}+A_{2} \cos \left[\frac{2 \pi(t-50400)}{A_{4}}\right],|t-50400|<A_{4} / 4 \\
5 \times 10^{-9}, \\
|t-50400| \geq A_{4} / 4
\end{array}\right.
$$

Where, $t$ is the local time at the intersection point of ionosphere connect with the satellite receiver (Range of values is $0 \sim 86400$ ). $A_{2}$ is the amplitude of the cosine curve for the daytime ionosphere delay, obtained by $\alpha_{n}$. $A_{4}$ is the period of cosine curve, obtained by $\beta_{n}$, which is detailed in references [4].

In the same way, $I_{Z}^{\prime}(t)$ represents vertical ionospheric delay, user have to multiply the inclination factor $F_{P P}$ to achieve the Inclined ionospheric delay.

\section{Grid Ionospheric Vertical correction error (GIVE) estimation}

Grid ionosphere vertical correction error (GIVE) is used to describe the accuracy of grid ionosphere delay, refers to the statistical analysis results of the difference value that the estimates value and the calculated value in a certain period of time. The specific calculation process is as follows:

(1) The master station uses ionospheric delay value of grid ionosphere received to calculate adjacent penetration point delay estimation $\hat{D}_{I P P}(t)$.

(2) The estimated values are compared with the calculated values of the value of duel frequency observation.

$$
e_{I P P}(t)=D_{I P P}(t)-\hat{D}_{I P P}(t)
$$

(3) The error limit can be obtained by statistical analysis of the penetration point of the ionosphere $e_{I P P}(t)$ in a certain period of time.

$$
\begin{aligned}
& E_{I P P}=\left|\bar{e}_{I P P}(t)\right|+k(\operatorname{Pr}) \sigma_{e} \\
& \left|\bar{e}_{I P P}(t)\right|=\frac{1}{n} \sum_{k=1}^{n} e_{I P P}(t) \\
& \sigma_{e}=\sqrt{\frac{1}{n-1} \sum_{k=1}^{n}\left(e_{I P P}(t)-\bar{e}_{I P P}(t)\right)^{2}}
\end{aligned}
$$

$K(\operatorname{Pr})$ represents the confidence level, which is 99.9\%.The GIVE value of the ionospheric penetration point is:

$$
G I V E=\operatorname{Max}\left\{E_{I P P, i}\right\}+\hat{e}_{I G P}
$$

$\hat{e}_{\text {IGP }}$ is behalf of the absolute error of the ionospheric delay of the lattice network, obtained by the the ionospheric delay error interpolation of penetration point. 


$$
\hat{e}_{I G P}=\frac{\sum_{j=1}^{n} W_{j}\left|e_{I P P}\right|}{\sum_{i=1}^{n} W_{i}}
$$

\section{Data processing and result analysis}

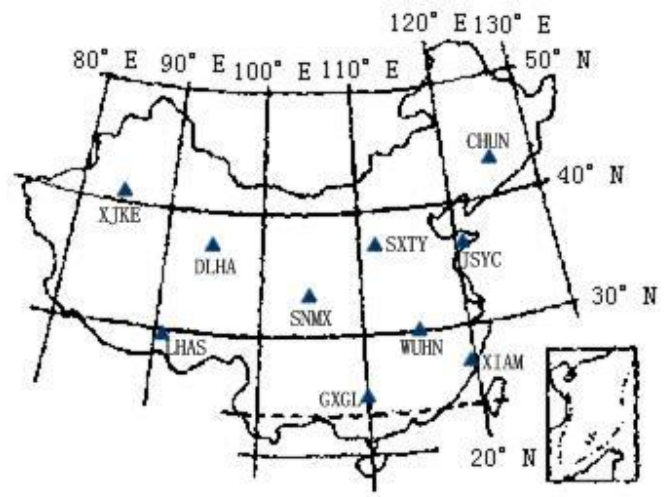

Fig. 3 The locations of ten GNSS reference stations in China

According to the algorithm of ionospheric delay model and grid ionosphere vertical error correction, based on the statistical analysis of the data of 10 GNSS stations in China in March 20, 2016 and the navigation message, the simulation of the Beidou satellite in the region of China was carried out.

\subsection{The comparison between grid ionospheric model and Klobuchar model of ionospheric delay correction}

Data acquisition time for the day of Beijing time 12 to 15, the sampling interval is $1 \mathrm{~s}$. taking the ionospheric delay of reference station in Wuhan (WUHN) and Taiyuan (SXTY) region as an example, respectively calculate the delay of penetration point with dual frequency algorithm and Klobuchar model algorithm and the algorithm of grid ionospheric. The results are shown in Figure 4 and figure 5.

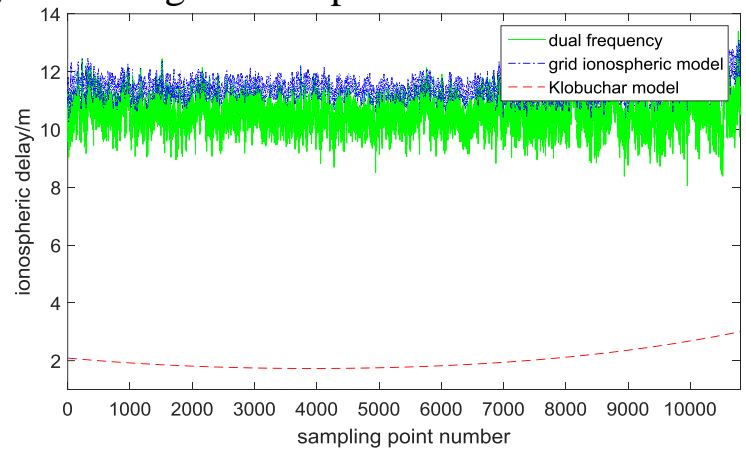

Fig. 4 The comparison among three model of ionospheric delay of WUHN

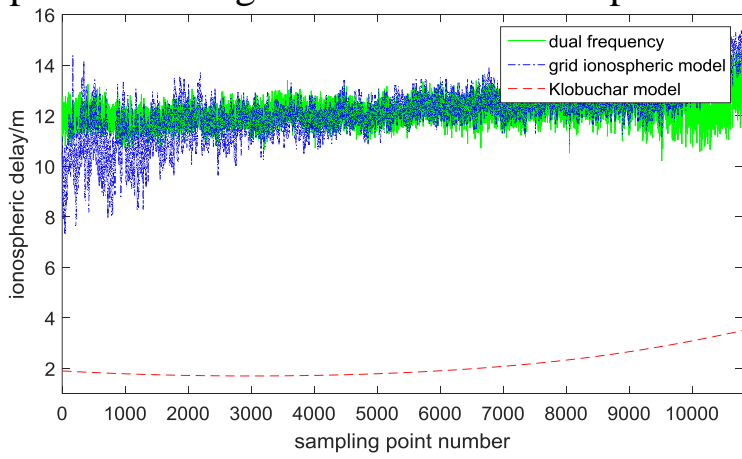

Fig. 5 The comparison among three model of ionospheric delay of SXTY 
Some data show that the dual frequency correction method can correct ionospheric delay correction more than $90 \%$. Compare the value for the correction of more accurate values to the dual frequency correction delay, from Figure 4 and figure 5 can be seen, the sampling time chosen for the day in the sun the most intense time, air molecules in the ionosphere in the decomposition of the most exuberant, ionospheric delay reached ten meters. Delayed Klobuchar model correction reachs 2 3m, so after the ionospheric delay of grid ionospheric model is better than the modified Klobuchar model of the ionosphere.

\subsection{Simulation results for GIVE}

Real time monitoring is carried out on the point of penetration of reference stations which are monitored at the observation time, the sampling period is $6 \mathrm{~min}$, and the calculated lattice sites and their GIVE values are calculated. Figure 6 and Figure 7 give the change of GIVE value in the observation time of 245 grid $\operatorname{dot}\left(27.5^{\circ} \mathrm{N}, 110^{\circ} \mathrm{E}\right)$ and $246 \operatorname{grid} \operatorname{dot}\left(32.5^{\circ} \mathrm{N}, 110^{\circ} \mathrm{E}\right)$.

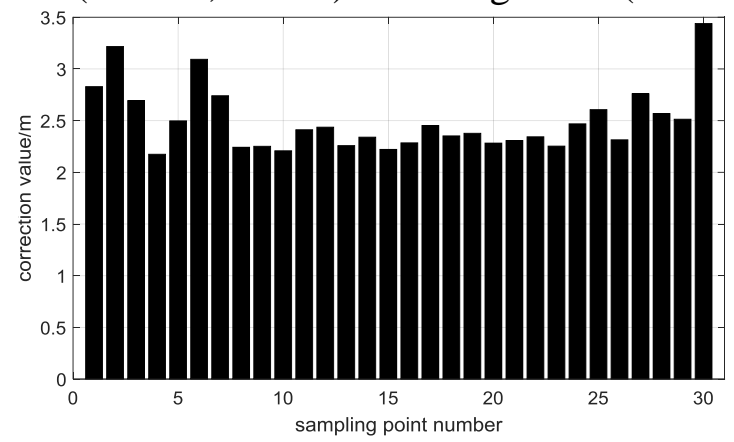

Fig. 6 GIVE of No.245 grid point

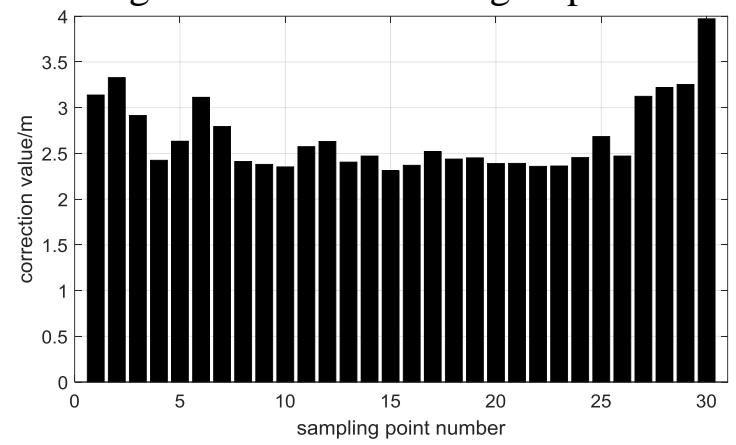

Fig. 7 GIVE of No.246 grid point

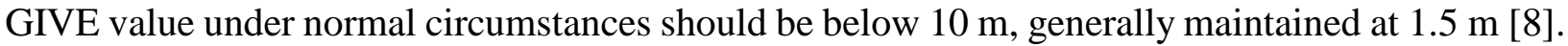
The GIVE value of the above two grid nodes is below $4 \mathrm{~m}$, and the value is not reached the ideal range. Through the analysis, the result is poor because the selection of the national reference stations is not enough, leading to the establishment of the ionosphere model is simple, cannot accurately calculate the ionospheric delay of grid points, and therefore cannot reflect the real situation.

\section{Summary}

This paper is based on the Beidou navigation system, the grid ionospheric model and the Klobuchar model is studied by using the 10 GNSS stations in the Beidou observation data. The verification results show that the grid ionospheric model for ionospheric delay correction effect is far better than that of Klobuchar model, BD grid ionospheric model proposed delay algorithm is feasible. The calculation of error correction of GIVE grid ionospheric model the result is not very ideal, result from the number of the reference station selected is not enough. If ionospheric grid model covers more reference station, it will provide the country's SBAS users with higher accuracy of the ionosphere correction and integrity of data.

\section{References}

[1] Xie Gang. Principle of GPS and receiver design. Beijing: Publishing House of Electronics 
Industry, 2009: 80-85.

[2] DO-229D (SC-159). Minimum operational performance standards for global positioning system/wide area augmentation system airborne equipment (WAAS) [S]. Washington, DC: RTCA Inc.,2006

[3] Force USA. NAVSTAR GPS space segment/navigation user interfaces [J]. Interface specification IS-GPS-200D, US Air Force, 2006, 3.

[4] BDS-SIS-ICD-2.0[S].BDS Management Office of China,2013

[5] Zhan Xianlong, Liu Ruihua, Yang Zhaoning. A study on the grid ionospheric delay algorithm in Beidou [J]. Aerospace Control, 2012, 30(1):15 19.

[6] Ormand D. Wide area augmentation system (WAAS) [R]. Raytheon System Co Fullerton CA, 1999.

[7] Wang Yongcheng, Huang Jianyu. Grid Algorithm of ionospheric delay in a GPS wide-area augmentation system [J]. Journal of China Institute of Communications, 1998, 19(12): 38-41.

[8] Cao Haiyang. Theory and technique research on GNSS integrity [D]. Xi'an: Chang'an University, 2013. 